\title{
Aprendizaje universitario en contexto de ASPO (Aislamiento Social Preventivo y Obligatorio)
}

\author{
University learning in the context ASPO (Social, Preventive and \\ Compulsory Isolation)
}

Natalia Soledad Petric*

\section{Resumen}

El artículo presenta una aproximación descriptiva a la experiencia estudiantil de aprendizaje universitario en contexto de ASPO (Aislamiento Social Preventivo y Obligatorio) debido a la pandemia por Covid-19. Para la misma, se confeccionó un formulario Google con preguntas -abiertas y cerradas- que se envió vía correo electrónico a los estudiantes de todas las carreras de la Facultad Teresa de Ávila UCA, Sede Paraná durante el mes de junio del corriente año. Fue respondido de manera voluntaria por 152 estudiantes de carreras correspondientes al Departamento Humanidades.

Se exponen una descripción general de los estudiantes que respondieron el cuestionario; los recursos con que cuentan para el cursado; el uso de la plataforma virtual EVA (Entornos Virtuales de Aprendizaje) y la experiencia de aprendizaje durante el ASPO. Esta última se categorizó según el aprendizaje de recursos tecnológicos, vinculares, autoorganización y autogestión. Además, se mencionan las dificultades con las que se encontraron, tales como cambios en las rutinas y cotidianeidad, relaciones con los docentes, el ser ingresante, la comunicación mediada por la tecnología y las habilidades digitales. Por último, los apoyos con los que cuentan y las demandas o necesidades para poder continuar con el cursado de manera no presencial.

Palabras claves: Aprendizaje universitario, Aislamiento social preventivo y obligatorio, Experiencia estudiantil

\begin{abstract}
This article presents a descriptive approach to the student experience of university learning in the context of ASPO (Preventive and Mandatory Social Isolation) due to the Covid-19 pandemic. For this, a Google form was made with questions -open and closedthat was sent via email to students of all careers of the Teresa de Ávila UCA Faculty, Paraná Headquarters during the month of June of this year. It was answered voluntarily
\end{abstract}

\footnotetext{
*Pontificia Universidad Católica Argentina Sede Paraná. Centro de Investigación Interdisciplinar en Valores, Integración y Desarrollo Social. Mail de contacto: nataliapetric@hotmail.com DOI: https://doi.org/10.46553/RPSI.16.32.2020.p104-118
}

Fecha de recepción: 19 de mayo de 2019 - Fecha de Aceptación: 11 de noviembre de 2019 
by 152 students from careers corresponding to the Humanities Department.

It presents a general description of the students who answered the questionnaire, the resources they have for the course, the use of the virtual platform EVA (Virtual Learning Environments) and the learning experience during the ASPO. The latter was categorized according to the learning of technological, bonding, self-organization and self-management resources. In addition, the difficulties they encountered are mentioned, such as changes in routines and daily life, relationships with teachers, being a newcomer, communication mediated by technology and digital skills and finally, the support they have and the demands or needs to be able to continue with the course in a remote manner.

Keywords: University learning, Preventive and compulsory social isolation, Student experience

\section{Introducción}

El presente artículo contiene la descripción de la información obtenida a partir del relevamiento acerca de la experiencia de aprendizaje universitario en contexto de Aislamiento Social Preventivo y Obligatorio (ASPO) debido a la pandemia por Covid-19, decretada por el gobierno nacional en el mes de marzo de 2020.

Esta situación se presentó como una ruptura en la cotidianeidad de todos los habitantes a nivel mundial y particularmente en el contexto argentino.

El contexto universitario no quedó ajeno a esta circunstancia. En este sentido el informe Unesco - Iesalc 2020 detalla que la pandemia está afectando en Latinoamérica a unos 23,4 millones de estudiantes universitarios y uno de los impactos a corto plazo que se registra en el caso de los alumnos es la reorganización de su vida cotidiana para ajustarse a una situación de confinamiento. "Inevitablemente, la pérdida de contacto social y de las rutinas de socialización que forman parte de la experiencia cotidiana de un estudiante de educación superior tendrán un costo" (Unesco, 2020, pag. 16)

Es por ello que se consideró de suma relevancia conocer la experiencia de los estudiantes universitarios ya que, al ser una vivencia sin precedentes, se constituye en sí misma en un objeto de estudio que amerita ser profundizado, para así generar dispositivos académicos e institucionales que coadyuven con mejoras en la calidad de los procesos de aprendizaje.

Los objetivos de esta investigación fueron, describir los recursos con que cuentan los estudiantes para el cursado virtual; indagar acerca del uso de la plataforma virtual EVA (Entornos Virtuales de Aprendizaje); y caracterizar las experiencias de aprendizaje de estudiantes universitarios en contexto de ASPO.

En este sentido se clarifica que EVA es el entorno virtual de aprendizaje que utiliza la Universidad Católica Argentina; similar a Moodle, aunque éste es un programa de código abierto. Este entorno virtual permite el uso de herramientas informáticas que posibilitan la interacción didáctica de manera que el estudiante pueda llevar a cabo las tareas de aprendizaje tales como comunicarse, leer documentos, realizar ejercicios, formular preguntas al docente, trabajar en equipo, etc. Todo ello de forma online, sin que medie una interacción física entre docentes y estudiantes (Area y Adell, 
2009).

La particularidad de su uso en el contexto de la UCA es que con anterioridad al ASPO, EVA era una herramienta complementaria al dictado de clases presencial, y a partir del ASPO, se convirtió en el recurso primordial y central para poder dar cumplimiento a la tarea educativa.

En este sentido se puede inferir que estos cambios en las modalidades de enseñanza y aprendizaje pueden generar reestructuraciones, acomodaciones, adaptaciones en las experiencias de aprendizaje de los y las estudiantes.

Indagar acerca de la experiencia estudiantil nos posiciona desde una perspectiva teórica y metodológica, que según Carli (2012) permite “... comprender los procesos nodales del devenir institucional, los procesos de identificación intra e intergeneracional y las dinámicas de la producción, trasmisión y apropiación del conocimiento en la universidad" ( $p$. 24). Implica atender a las prácticas de los sujetos y sus reflexiones, y poner en cuestión una "mirada generalista" (Carli, 2012) para conocer los modos en que los estudiantes transitan la vida universitaria.

\section{Metodología}

Muchas universidades durante este tiempo construyeron diferentes formas de relevar la experiencia de estudiantes (Gandulfo, Alegre y Domínguez, 2020; Lozano-Díaz, Fernández-Prados, Figueredo Canosa y Martínez Martínez, 2020) y de docentes (De Vincenzi, 2020; Finkelstein, 2020; Federación de docentes de la Universidad, 2020.

Con este objetivo se confeccionó un formulario ad hoc en Google con preguntas -abiertas y cerradas- que se envió vía correo electrónico a los estudiantes de todas las carreras de la Facultad Teresa de Ávila UCA Sede Paraná durante el mes de junio del corriente año.

El cuestionario, se estructuró en cinco secciones, las cuatro primeras con opciones cerradas de elección: a) Datos generales -sexo, carrera, año de cursado-; b) Recursos tecnológicos -tipos, uso de los dispositivos, conexión a Internet, uso de plataformas, autocalificación de su uso-; c) Modalidad de estudio; d) Uso de EVA -frecuencia, dificultades, aspectos positivos y negativos, comunicación con docentes, antecedentes en su utilización- y uso de la biblioteca virtual y e) Experiencias de aprendizaje en cuarentena: preguntas abiertas acerca de los aprendizajes en este contexto, de las dificultades que se les presentaron y a los apoyos obtenidos y solicitados.

Al no haber precedentes de estudio en el contexto estudiado, se constituye el estudio, en exploratorio y descriptivo.

El cuestionario fue respondido de manera voluntaria, por 152 estudiantes de carreras correspondientes al Departamento Humanidades, quedando la muestra conformada de la siguiente manera:

Respecto del sexo de los estudiantes que respondieron el cuestionario, el $92 \%$ es de femenino.

Con relación a la carrera de pertenencia en porcentajes similares, los participantes cursan la Licenciatura en Psicopedagogía $(36,8 \%$ ) y la Licenciatura en Psicología (36,2\%), en tercer lugar (21\%) fueron estudiantes de la Tecnicatura en Bibliotecología y por último $(5,9 \%)$ del Profesorado en Educación Superior. 

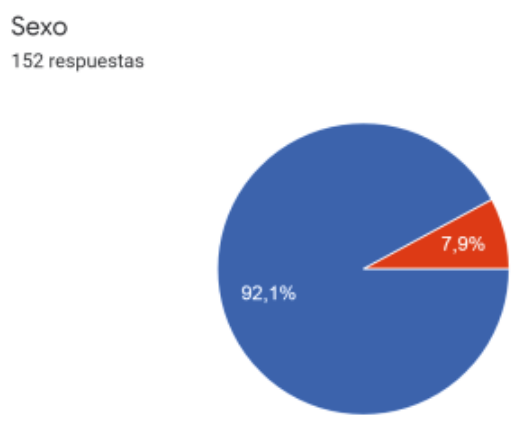

Figura 1. Distribución de estudiantes por sexo

Carrera que esta cursando

152 respuestas

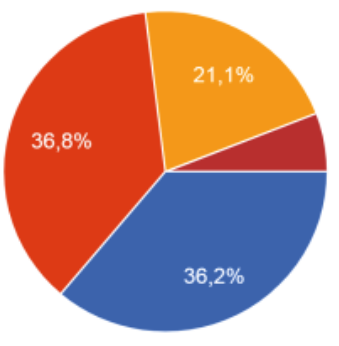

Lic. Psicología

Lic. Psicopedagogía

Bibliotecología

Abogacía

Lic. Relaciones Internacionales

Contador Público

Martillero Público

Notariado

$1 / 2 \nabla$

Figura 2. Distribución de estudiantes por carrera de pertenencia

La mayoría de los estudiantes que respondieron el cuestionario $(36,2 \%)$ cursan el primer año; el 28,3\% cursa el tercer año; el $27 \%$ cursa segundo año; y entre un $6,6 \%$ y un $2 \%$ pertenecen al cuarto y quinto año respectivamente.

El análisis de las preguntas cerradas se realizó a partir de estadística descriptiva básica -frecuencias y porcentajes- y el análisis de las preguntas abiertas se planteó desde la conformación de categorías de análisis generales, bajo el criterio de maximización de similitudes.

\section{Resultados}

A continuación, se describen los resultados, organizados en tres secciones:

1. Recursos para el cursado.

2. Uso de EVA (Entornos Virtuales de Aprendizaje). 
Año que actualmente estas cursando (Si cursas más de un año, consigná el que tenga mayor carga de materias)

152 respuestas

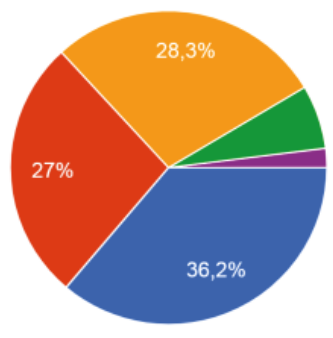

Primer año

Segundo año

Tercer año

Cuarto año

Quinto año

Figura 3. Distribución de estudiantes por año de cursado

En la actualidad: ¿contás con algún dispositivo tecnológico para poder ingresar a las aulas virtuales?

152 respuestas
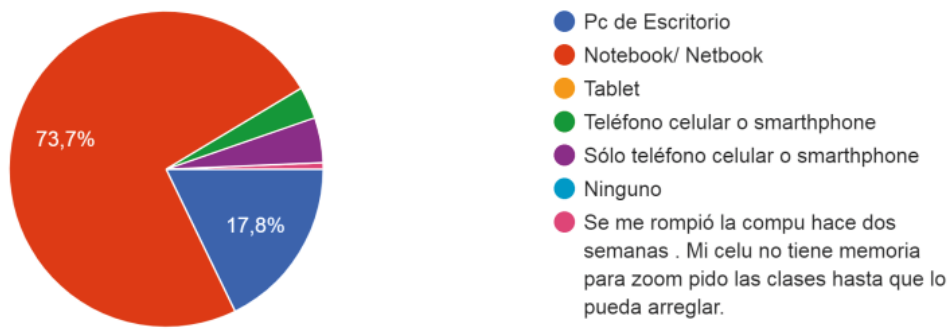

Figura 4. Distribución de acceso a dispositivos tecnológicos

3. Acerca de la experiencia de aprendizaje durante el ASPO.

\section{Recursos materiales para el cursado}

Respecto de los recursos materiales -principalmente dispositivos tecnológicos y conectividad- para que el cursado sea factible, se encontró que el $73 \%$ cuenta con Notebook/Netbook, un $17,8 \%$ cuenta con $\mathrm{PC}, \mathrm{y}$ el resto cuenta con teléfono celular.
Respecto de la pregunta acerca de si estos dispositivos son de uso individual o compartido, los porcentajes son similares para quienes debe compartir $(46,7 \%)$, como para quienes tienen uso exclusivo $(53,3 \%)$. Este aspecto es importante en cuanto la disponibilidad de recursos, se relaciona con el tiempo del que disponen los estudiantes para estar conectados exclusivamente a sus clases y demás actividades académicas.

Además de los dispositivos, es 
Estos dispositivos ¿ Los compartis con alguien más?

152 respuestas

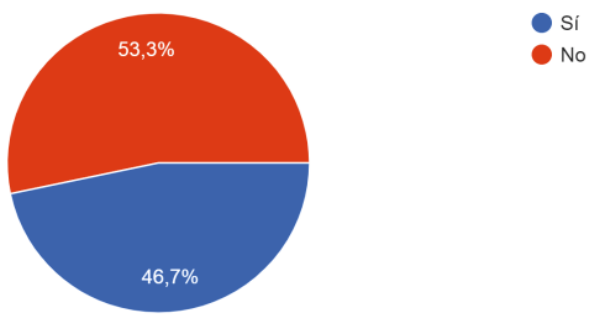

Figura 5. Distribución respecto de si comparten o no los dispositivos tecnológicos

En la actualidad ¿ Contas con conexión a internet?

152 respuestas

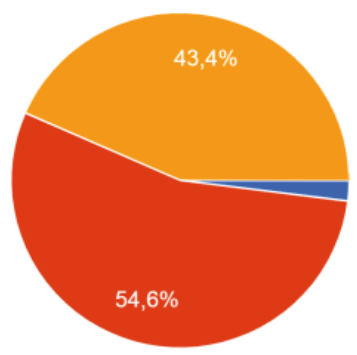

Cuento únicamente con datos del celular con abono fijo o prepago

Cuento con wifi del servicio de mi hogar

Cuento con datos móviles y wifi

Figura 6. Distribución respecto al tipo de conectividad a Internet

necesario contar con conexión a Internet para poder acceder a clases. La mayoría $(54,6 \%)$ cuenta con conexión WiFi de su hogar y el 43,4\% cuenta con datos móviles más conexión WiFi. Sólo un $2 \%$ cuenta únicamente con datos del celular.

El escaso porcentaje de estudiantes que solo cuentan con datos móviles del celular posiciona al grupo indagado positivamente respecto de enorme brecha digital entre países y dentro de cada país respecto de la posibilidad de tener acceso a Internet, aunque en Argentina las tasas de líneas móviles son extremadamente elevadas y superan, en muchos casos, la cifra de una línea por persona (Unesco, 2020).

A la indagación acerca de los recursos tecnológicos más utilizados en este tiempo de ASPO, los estudiantes respondieron que la plataforma más utilizada es Whatsapp, lo sigue EVA, luego el Correo electrónico, Instagram $\mathrm{y}$, por último, pero con uso 
¿Has usado o tenes acceso a estos recursos tecnológicos?

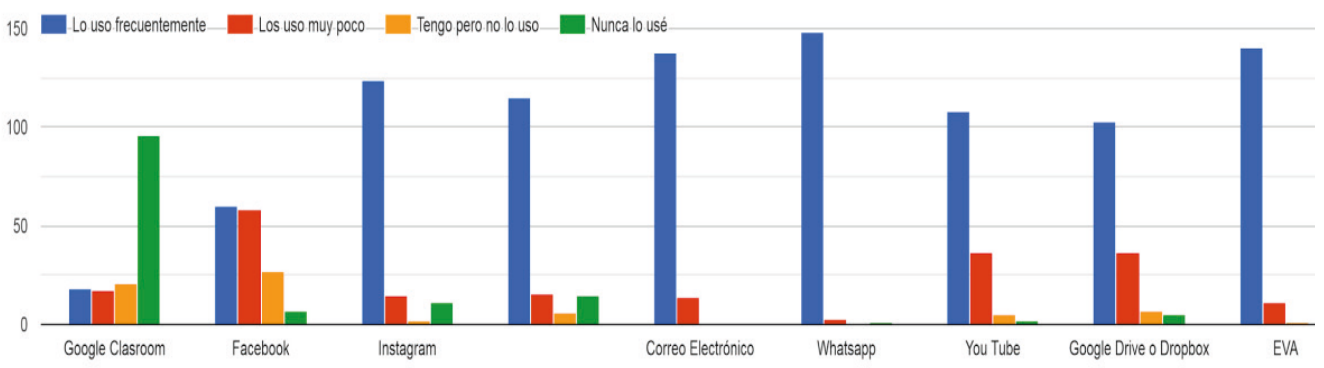

Figura 7. Distribución respecto del uso de recursos tecnológicos

¿Cuál considera que es tu manejo frente al uso de plataformas y aplicaciones tecnológicas utilizadas en el aprendizaje?

152 respuestas
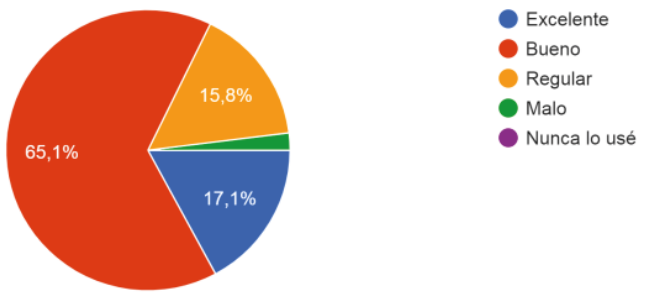

Figura 8. Distribución del grado de experticia en el uso de recursos tecnológicos

frecuente ZOOM, Meet. La plataforma menos utilizada es Google Clasroom.

Respecto del grado de experticia en el uso de las mencionadas plataformas, los estudiantes expresaron que el manejo es "Bueno" (65,1\%), "Excelente" (17,1\%) y en tercer lugar un uso "Regular" $15,8 \%$. Este aspecto destaca las habilidades digitales de los y las estudiantes (Caragliano, 2015).

Respecto de la modalidad de estudio, el $62,5 \%$ estudia solo y solo un $29,6 \%$ en conexión con otros compañeros.
Interesante aspecto para intervenir psicoeducativamente, estimulando la "no pérdida" del capital social virtual o capital social online (Nguyen, Dao, Phung, Venkatesh y Berk, 2013).

\section{Uso de EVA (Entornos Virtuales de Aprendizaje)}

Con relación al uso de la plataforma propia de la Universidad (EVA), los estudiantes manifestaron no tener dificultades 
Frente al cursado virtual ¿ Cómo estudias?

152 respuestas

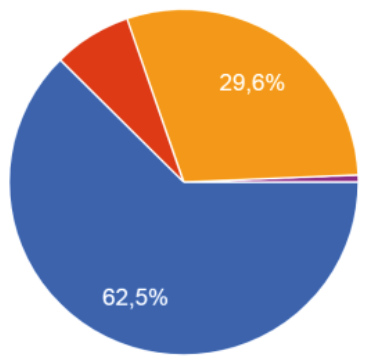

Solo/a

Algún/a compañero/a te ayuda en las tareas y actividades digitales que se te proponen

Te conectas con amigos/as o compañeros/as para llevar adelante actividades de lectura y/o estudio

Con familiar

Mayoritariamente sola, a veces con familiar

Figura 9. Distribución respecto de la modalidad de estudio

¿Haz podido matricularte en EVA a todas materias que estas inscripto?

152 respuestas
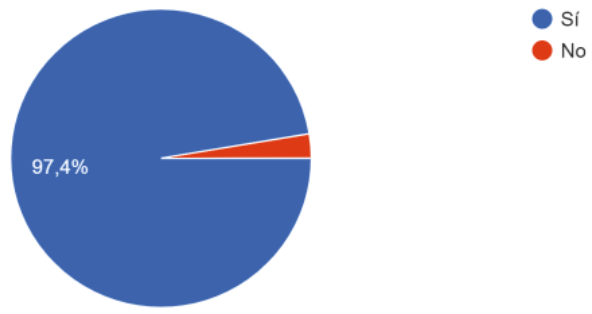

Figura 10. Distribución respecto de dificultades de matriculación en EVA

con la matriculación en las aulas y materias cursadas:

Respecto del uso de la plataforma, un $67,8 \%$ manifiesta no haber tenido dificultades y un $20,4 \%$ expresa haber tenido diferentes inconvenientes especialmente de conectividad y demoras en la posibilidad de matricular por parte de la Facultad.

Además, se consultó si durante la presencialidad, EVA era utilizada en las cátedras. El resultado muy similar entre el porcentaje de los que si la utilizaban $(48,7 \%)$, como el de los que no utilizaban $\operatorname{EVA}(51,3 \%)$.

Las respuestas acerca de los aspectos positivos del uso de EVA remiten: al fácil acceso y uso intuitivo; a la organización de las materias -desde la disponibilidad del material de cátedra hasta subir los trabajos prácticos-; a la rapidez en la devolución de calificaciones; al uso exclusivamente académico; y a la economía que permite el contar con el material digital.

Asimismo, los aspectos negativos incluyen: que algunos docentes no saben utilizarla, lo que se presenta como un obstáculo; la falta de notificaciones en los movimientos del aula; dificultades de conectividad; el exceso de material y de horas de cursado virtual y el que la plataforma no guarda trabajos de años anteriores. 
Durante el dictado de clases presenciales, ¿en alguna/s cátedras empleaban la plataforma EVA? 152 respuestas

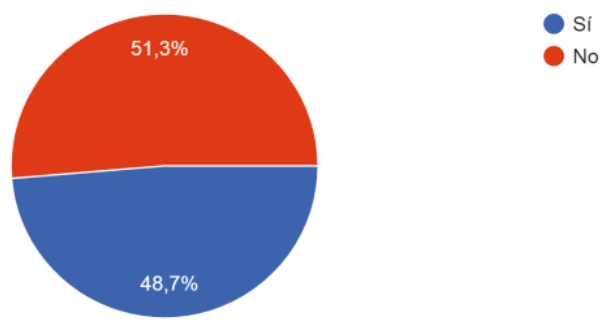

Figura 11. Distribución de uso previo al ASPO de EVA

¿Cómo te resulta la comunicación e intercambio con el docente desde esta plataforma?

152 respuestas

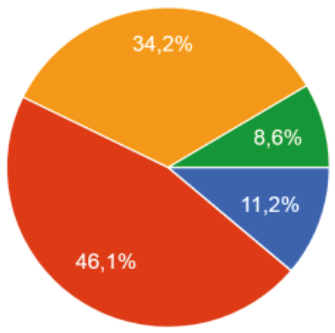

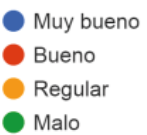

Malo

Figura 12. Distribución respecto de la comunicación con docentes a través de EVA

Este aspecto podría comprenderse en el sentido que las cátedras no fueron pensadas originalmente para ser dictadas con modalidad virtual, lo que genera desorganizaciones, escasa selectividad de material y recursos que se plantean como una solución paliativa al contexto emergente (De Vincenzi, 2020), además que las expectativas de los estudiantes son distintas si esperan matricularse, desde el principio, en un curso de educación a distancia o bien en un curso presencial, con todos los elementos sociales y experienciales que acompañan siempre la experiencia presencial (Unesco, 2020).

Ya con respecto a la comunicación con los docentes, mediada por la virtualidad y la plataforma, los estudiantes manifestaron que fue "Buena" en un 46,1\% y "Regular" en un $36,2 \%$.

Como otro recurso de utilidad para el cursado, se consultó sobre el uso de la biblioteca virtual de la universidad, es así que un porcentaje mayoritario expresó no utilizarla $(68,4 \%)$. Este aspecto deberá ser considerado por la Universidad para ser difundido en su utilización y modos de acceso.

\section{Acerca de la experiencia de aprendizaje durante el ASPO}

Las experiencias de aprendizaje se 


\section{¿Utilizás el recurso de la biblioteca virtual para consultar bibliografia de las diversas materias?} 152 respuestas

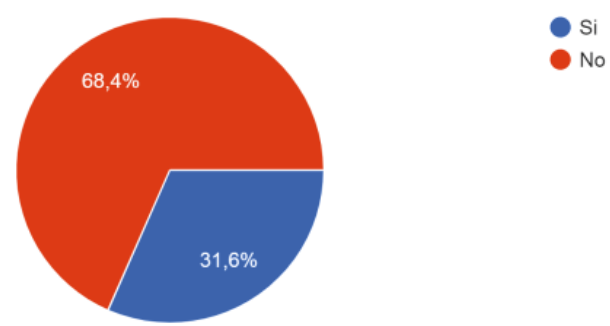

Figura 13. Distribución respecto de la utilización de la biblioteca virtual de la universidad

categorizaron en tres ejes: aprendizajes en contexto o recuperados por los estudiantes; dificultades devenidas; y apoyos actuales y necesitados. A continuación, se expande sobre ellos mediante testimonios representativos de los estudiantes.

\section{Aprendizajes en contexto}

Las subcategorías que dan cuenta de estos fueron: el manejo de recursos tecnológicos; nuevos vínculos; autoorganización y autogestión; y valorar la presencialidad.

El aprendizaje acerca del manejo de recursos tecnológicos se manifestó de la siguiente manera:

"Utilización de drive (para presentaciones, o documentos compartidos), utilizar la biblioteca digital más seguido, utilizar diferentes plataformas digitales (EVA, ZOOM)".

"Usar la plataforma, armar trabajos prácticos por zoom, grabar las clases y armar mis apuntes."

El aprendizaje de nuevos vínculos, a partir de nuevas maneras de relacionarse con pares, docentes y familiares:

"Aprendí a concentrarme y estudiar rodeada de otras personas realizando actividades diversas (...) a trabajar de manera virtual con mis compañeros." "Más allá de los aprendizajes teóricos referidos a cada materia, remarcaría el hecho de haber desarrollado más la perseverancia y la empatía, la utilización de páginas webs para resolver diferentes actividades de las cátedras realizado infografías o presentaciones."

Y la autoorganización y autogestión del aprendizaje se expresó de la siguiente manera:

"A familiarizarme más con la tecnología, buscar artículos en la computadora y ser más independiente".

"Responsabilidad, manejo del tiempo y organización del estudio".

"Adaptarme a una nueva forma de interactuar en las clases, organizarme de diferente manera en cuanto a los horarios de estudio 
y/o actividades, estar más pendiente de los comunicados por parte de la universidad."

Algunos estudiantes resaltaron el añorar, y así valorar la presencialidad:

"Aprender a valorar la presencialidad y la riqueza del contacto humano."

\section{Dificultades encontradas}

Por parte de los estudiantes durante este período, fueron principalmente las devenidas de: la situación de ASPO; cuestiones docentes; el ser ingresante; la comunicación mediada por la tecnología; y el estudio con principal soporte digital.

Con relación a la situación de ASPO, los estudiantes manifestaron dificultades de concentración, atención, vinculación y adaptación a un nuevo ritmo y contexto:

"Al principio resultó muy difícil compartir mi espacio de estudio con otras personas".

"Falta de atención ya que no es lo mismo estar en tu casa que en la facultad".

"Me costó mucho más estudiar que antes ya que al estar toda mi familia a la mañana (cosa que no sucedía en la normalidad), hay muchos distractores en la casa que interrumpen el tiempo de estudio".

"No saber cómo organizarse y la incertidumbre".

"Mi mayor dificultad es sostener la atención y concentración (poco entrenada) por mucho tiempo frente a la computadora, me costó sostener el ritmo y perseverancia en el cursado. $\mathrm{Me}$ es dificultoso recibir la clase expositiva sin poder luego retomar dudas o inquietudes debido a la falta de tiempo para tener las lecturas al día."

"Que es muy cansador al estar encerrados, que falta la dinámica con pares y profesores, cuesta más aprender de esta manera a mi parecer".

"Tiempo, para interpretar las unidades de las 8 cursadas. Trabajo. La familia y las tareas del hogar, higiene; se multiplicó en todos los aspectos."

Otra dificultad señalada fue referida a cuestiones docentes:

"No siento que el personal docente esté capacitado para organizarse en esta plataforma, las formas de evaluación no me parecen correctas para la carrera (multiple choice), los profesores no dan el espacio de aclarar dudas ni podemos saber en qué nos equivocamos."

"El no compromiso de algunos docentes de facilitarnos el material a tiempo o contestar las dudas acerca de los trabajos que mandan. Y falta de programas en algunas cátedras."

El ser ingresante, como primera experiencia en este ámbito fue resaltado como dificultad:

"Es mi primer año por lo que no logro entender el mecanismo de la facultad ya que nadie informa con precisión. $\mathrm{Y}$ por eso mismo tengo problemas en la organización ya que no tengo experiencia en la vida universitaria." "Al ser de primer año creo que también nos cuesta más, ya que necesitamos el día a día en la facu para sentirnos más acompañados por todos y más seguros con toda esta vida universitaria que vamos descubriendo y a la cual nos acostumbramos de a poco."

"Al principio no entendía nada porque 
era algo nuevo para mí, tanto la vida universitaria como las materias y sus temas. No es lo mismo la explicación de 1 hora tras la pantalla, que ocupar todas las horas en una clase presencial. No hay confianza ya que no conozco bien a los profesores y a veces me da vergüenza preguntarles cosas. Yo tuve la suerte de conocer chicos e hice unos amigos, pero muchos no tuvieron esa oportunidad o hicieron el ingreso directo por internet, así que a la hora de hacer grupos puede ser complicado."

Las dificultades de comunicación mediada por la tecnología fueron señaladas también:

"Otra dificultad del cursado fueron los errores de transmisión de información sobre qué es lo que había que hacer en determinados trabajos prácticos. Las consignas eran muy vagas, lo que dio lugar a contradicciones".

Dificultades por falta de hábito respecto del estudio básicamente con soporte digital:

"Se me dificultó poder leer todo el material que nos han enviado, ya que muchas veces este no era nítido y debías esforzar mucho la vista para poder leerlo."

"Aprendo leyendo, me cuesta concentrarme mucho más, las clases son buenas, pero a veces muy pesadas ya que algunas son prácticas y es difícil seguir el hilo a través de zoom. También otras materias que por tema de pandemia no tuvimos casi clases."

\section{Apoyos actuales y necesitados}

Cuando se presentan dificultades durante el cursado y el estudio, los estudiantes acuden principalmente a sus compañeros, a sus familiares y al área de Orientación de la Facultad. Algunos también cuentan con apoyo terapéutico particular (psicólogo).

Por último, con relación a la consulta acerca de las cuestiones que los estudiantes consideran sería de ayuda para continuar con su proceso de aprendizaje universitario en este contexto de cuarentena, se resaltó la necesidad de flexibilizar evaluaciones, materiales de clase, trabajos solicitados:

"La posibilidad de poder promocionar alguna materia del plan de estudio a través de un trabajo integrador de los contenidos desarrollados y exposición del mismo."

"Menos exigencia y más contemplación por parte de los profesores e instituciones. Que entiendan las situaciones de cada uno, sean más contemplativos en su justa medida."

"Que los profesores se adapten a la plataforma y los contenidos en digital. Si no está la bibliografía que usan siempre que la reemplacen por otra en digital. No pedimos menos material o exigencia sino flexibilidad."

"Que algunos profesores estén más atentos a nuestras necesidades. Que haya mayor comunicación y se reduzca lo más posible las incertidumbres respecto al cursado, trabajos y exámenes."

Y también la diversificación de estrategias didácticas a la hora del dictado de clases:

"Que las clases no sean solo expositivas y se empleen diferentes recursos virtuales que atiendan a los 
diferentes estilos de aprendizaje que tenemos los estudiantes. Por ejemplo: en zoom, pasar Powers con imágenes, sonidos, etc."

"Trabajos prácticos o tareas para que el material no quede en una simple lectura y estemos más interiorizados en los temas ya que con la poca carga virtual quedan los temas medios superficialmente dados."

"Clases más explicativas para lograr el entendimiento y que realmente las cosas queden en nosotros y no exceso de trabajos prácticos."

\section{Conclusión}

Es importante resaltar que los estudiantes que respondieron el cuestionario expresaron contar con recursos para acceder a la cursada mediada por la tecnología, ya que cuentan con dispositivos tecnológicos y con conectividad, tanto WiFi como datos móviles. Este aspecto los posiciona favorablemente frente a otras realidades en las que las dificultades de conectividad pueden constituirse en factores de deserción.

La plataforma EVA logró posicionarse como recurso central de uso para organizar el cursado, como también el uso de Whatsapp para un uso más informal.

A pesar de que solo un poco menos de la mitad de los estudiantes expresaron haber utilizado EVA con anterioridad al ASPO, no generó dificultades en su implementación.

Además, los estudiantes se auto perciben desde una experiencia buena y excelente en el uso de estos recursos, aspecto que resalta las habilidades digitales de los mismos, aunque en relación con los docentes, es necesario profundizar en la enseñanza de estas habilidades, que no podrán quedar supeditadas a un aprendizaje individual y personal, sino atravesar a toda la comunidad. En este sentido es fundamental profundizar en estas habilidades, especificándose en sus dominios: operacional, formal, de información y estratégico (Van Deursen, Helsper y Eynon, 2014).

Respecto del capital social, la mayoría estudia solo, pero esto puede verse influido porque un alto porcentaje de estudiantes son ingresantes, por lo que no han podido aún consolidar grupos de estudio.

La población de ingresantes -en el caso de provenir desde la escuela secundariase tornaría más vulnerable que el resto dado que no cuenta con referencias del contexto universitario (Unesco, 2020). Como también los estudiantes prontos a graduarse, ya que las perspectivas laborales son inciertas.

Los principales aprendizajes remiten a usos tecnológicos diversos, nuevos modos de vincularse con sus contextos inmediatos y con sus compañeros y aprender modos de autogestionar y auto organizar su aprendizaje de manera más autónoma que en el cursado presencial.

A su vez, las dificultades se centralizan en la adaptación que requiere la ASPO respecto de contextos de convivencia, modos de compartir espacios y recursos, gestionar las emociones de incertidumbre, cansancio y ansiedad. Algunos estudios señalan el fuerte impacto que el confinamiento ha tenido sobre la situación vital del estudiantado en el ámbito psicológico y académico, explicando que los estudiantes tienen más problemas psicológicos o pedagógicos que técnicos (Lozano-Díaz et al., 2020). 
Otra dificultad manifestada es la comunicación con docentes y hábitos de estudio basado exclusivamente en materiales digitales. En este sentido se puede cuestionar en cierta medida la idea de "los nativos digitales" -estudiantes nacidos en la era de las tecnologías digitales-, dado que los mismos pueden llegar a tener menos dificultades para el acceso, pero la idea de que aprenden más y mejor solo por implementar dispositivos de aprendizaje con la última tecnología disponible es simplista, ya que el uso para objetivos académicos requiere de habilidades digitales (Burin, Coccimiglio, González y Bulla, 2016).

Por último, lo que los estudiantes expresan necesitar para sostener su cursado, es un contexto flexible, que contemple la inestabilidad del tiempo vivenciado y la ansiedad que generan las adecuaciones $\mathrm{y}$ adaptaciones recurrentes a esta nueva experiencia que transitan, como también diversificar las estrategias didácticas que permitan acceder a los contenidos desde diferentes estilos aprendizaje.

En este sentido es interesante considerar que el perfil profesionalizante que tiene la universidad, es decir formar profesionales que se desempeñarán en diferentes campos, implica el aprendizaje de contenidos altamente especializados y orientados hacia la formación en una profesión de carácter científico, tecnológico o artístico (Finkelstein, 2020). Esto demanda el diseño de recursos didácticos y pedagógicos consonantes con el contexto y con el fin formativo, sin dudar que las lecciones aprendidas serán un capital de conocimiento que, en las modalidades híbridas de educación que se plantean para el retorno a la presencialidad, serán de gran utilidad.

Por último, y en sintonía con lo expuesto por Unesco (2020) "se debe considerar que los efectos presentes de la crisis sobre la educación superior son fácilmente documentables, pero aquellos que dejarán huella en los distintos actores a medio y a largo plazo resultan más sujetos a debate" (pag.9).

Esta falta de referencias convoca a todos los actores institucionales a pensar, diseñar, proponer estrategias didácticas, pedagógicas y de contención y promoción emocional, para así comprometerse con los destinatarios últimos de la educación: los y las estudiantes.

\section{Referencias}

Area, M. y Adell, J. (2009). -eLearning: Enseñar y aprender en espacios virtuales. En J. De Pablos (Coord): Tecnología Educativa. La formación del profesorado en la era de Internet. Aljibe, Málaga, 391-424.

Burin, D., Coccimiglio, Y., González, F., y Bulla, J. (2016). Desarrollos recientes sobre Habilidades Digitales y Comprensión Lectora en Entornos Digitales. Psicología, Conocimiento y Sociedad, 6(1), 191-206. http://www.scielo. edu.uy/scielo.php?script $=$ sci a r t t e x t \& p i d = S 1688 70262016000100009\&lng=es\&tln $\mathrm{g}=$ es. 
Caragliano, E. (2015). Nuevas competencias digitales. Reflexión Académica en Diseño y Comunicación (XXVI) 89- 92. https://fido.palermo.edu/ servicios_dyc/publicacionesdc/ vista/detalle_articulo.php?id libro $=544 \&$ id_articulo $=11402$

Carli, S. (2012) El estudiante universitario. Hacia una historia del presente de la educación pública. Siglo Veintiuno.

De Vincenzi, A. (2020). Del aula presencial al aula virtual universitaria en contexto de pandemia de COVID-19. Avances de una experiencia universitaria en carreras presenciales adaptadas a la modalidad virtual. Debate Universitario, 8(16), 67-71. http://200.32.31.164:9999/ojs/ index.php/debate-universitario/ article/view/238/242

Federación de docentes de la Universidad (2020). Informe: el impacto de la virtualización en la educación universitaria.https://fedun.com. ar/informe-sobre-el-impactode-las-cursadas-virtuales-en-laeducacion-universitaria/

Finkelstein, C. (2020). La enseñanza en la universidad en tiempos de pandemia. Citep. Centro de Innovación en Tecnología y Pedagogía. [Sitio web] http://citep. rec.uba.ar/covid-19-ens-sin-pres/

Gandulfo,C., Alegre, T. y Domínguez, M. (Comps.)(2020). El ASPO en Primera Persona. Relatos de estudiantes universitarixs viviendo la cuarentena en el nordeste argentino. Colectivo Syry
Ediciones.

Lozano-Díaz, A., Fernández-Prados, J.S., Figueredo Canosa, V. y Martínez Martínez, A.M. (2020). Impactos del confinamiento por el COVID-19 entre universitarios: Satisfacción Vital, Resiliencia y Capital Social Online, International Journal of Sociology of Education, Special Issue: COVID-19 Crisis and Socioeducative Inequalities and Strategies to Overcome them, 79-104. http://doi.org/10.17583/ rise. 2020.5925

Nguyen, T., Dao, B., Phung, D. Q., Venkatesh, S., \& Berk, M. (2013). Online social capital: mood, topical and psycholinguistic analysis, in ICWSM 2013: Proceedings of the 7th AAAI International Conference on Weblogs and Social Media, AAAI Press, Palo Alto, Calif.,449-456.http://hdl.handle. net/10536/DRO/DU:30057170

Unesco. Instituto Internacional para la Educación Superior de América Latina y el Caribe (IESALC) (2020) COVID-19 y educación superior: De los efectos inmediatos al dia después. Análisis de impactos, respuestas politicas y recomendaciones.http://www. iesalc.unesco.org/wp-content/ uploads/2020/05/COVID-19ES-130520.pdf

Van Deursen, A. J. A. M., Helsper, E. J., y Eynon, R. (2014). Measuring digital skills. From digital skills to tangible outcomes. Project Report. http://www.oii.ox.ac.uk/research/ projects $/$ id $=112$ 